\title{
ADHS bei Erwachsenen
}

\section{Sulbstanzmissbrauch håufige Komorbiditåt}

- Auf der 6. ADHD Europe Conference in Berlin diskutierten renommierte Experten aktuelle Erkenntnisse zur Diagnostik und Therapie der ADHS. Ein Thema war der Substanzmissbrauch („substance use disorder“, SUD) bei erwachsenen Patienten.

Nach Professor Michael Huss, Direktor der Klinik für Kinder- und Jugendpsychiatrie und -psychotherapie der Johannes Gutenberg-Universität in Mainz, beobachtet man bei Kindern mit ADHS ein deutlich erhöhtes Risiko für Substanzmissbrauch in der Adoleszenz und im Erwachsenenalter. Es gebe allerdings Hinweise darauf, dass die Gefahr für Substanzmissbrauch durch die frühzeitige medikamentöse Therapie, beispielsweise mit retardiertem Methylphenidat, reduziert werden könne.

Umgekehrt zeige sich bei vielen Erwachsenen mit SUD eine klare ADHS-Historie in ihrer Kindheit. Allerdings gebe es kaum Langzeitstudien zu dieser Thematik, bedauerte Huss. Der National Comorbidity Study zufolge sind etwa $15 \%$ der erwachsenen ADHS-Patienten von SUD betroffen [Kessler RC et al., Am J Psych 2006 163 (4): $716-23$ ].
In jedem Fall ist laut Huss zunächst die vorherrschende Störung - ADHS oder SUD - zu behandeln, wobei die verschiedenen Therapieoptionen miteinander kombiniert werden sollten. Gleichzeitig muss bei Substanzmissbrauch vorsichtig mit der medikamentösen Therapie umgegangen werden: Vor Beginn einer Therapie der ADHS mit Methylphenidat (MPH, Medikinet ${ }^{\star}$ adult) sollte eine Suchtmittelabstinenz gegeben sein. Bei einem Rückfall im Therapieverlauf hält er eine Weiterbehandlung mit MPH für sinnvoll. Um die Adhärenz der Patienten zu erhalten, können bei guter Zusammenarbeit der Therapeuten anstelle von starren Regimen flexible Dosierungen von Vorteil sein, so der Experte. Vor dem Hintergrund, dass bei mehr als der Hälfte der ADHS-Kinder die Erkrankung im Erwachsenenalter persistiert, hält Huss die Erstellung eines Transitionsplans im Alter von etwa 14 Jahren für enorm wichtig [Turgay A et al., J Clin Psychiatry 201273 (2): 192 - 201].

Dr. Claudia Bruhn

„ADHD Europe Conference 2012", Berin, 21.4.2012

Veranstalter: MEDICE Arzneimittel, Pütter GmbH \& Co. KG

\section{Antidepressive Therapie}

\section{Die Schlüisselsymptome der Depression angehen}

- Das Antidepressivum Agomelatin (Valdoxan ${ }^{\circledR}$ ) kann neben der depressiven Stimmung auch Anhedonie, Angstsymptome und zirkadiane Rhythmusstörungen bessern. Besonders ausgeprägt ist die antidepressive Wirkung der Substanz bei Patienten mit schweren Depressionen.

Anhedonie ist ein Kernsymptom der Depression. Für die betroffenen Patienten ist das Phänomen mit Beeinträchtigungen im täglichen Leben verknüpft, berichtete Professor Massimo Di Giannantonio, Chieti, Italien. Um die Patienten in Remission zu bringen und spätere Rückfälle zu vermeiden, sei es wichtig auch dieses Symptom einer Depression effektiv zu behandeln.

\section{Antianhedone Wirkung von Agomelatin}

Die Ergebnisse einer aktuellen Studie mit 60 depressiven Patienten zeigen, dass Agomelatin sich auch bei der Linderung der depressionstypischen Anhedonie bewährt. Im Rahmen der Studie erhielten jeweils 30 Patienten über acht Wochen randomisiert Agomelatin $(25-50 \mathrm{mg} / \mathrm{d})$ oder Venlafaxin XR $(75-150 \mathrm{mg} / \mathrm{d})$. Zur Erfassung des Schweregrades der Anhedonie wurde die Snaith-Hamilton Pleasure Scale (SHAPS) verwendet, ein Fragebogen bestehend aus 14 Items, die jeweils eine potenziell angenehme oder genussvolle Aktivität beschreiben. Der Wertebereich des SHAPS liegt zwischen
0 und 14 Punkten, wobei höhere Werte eine stärkere Anhedonie repräsentieren. Agomelatin führte zu einer schnellen und signifikanten Reduktion des SHAPSScores. Dieser Effekt war von einer deutlichen Verbesserung des klinischen Gesamteindrucks (CGI) um 2,94 Punkte gegenüber Baseline begleitet $(p<0,05)$. In der Vergleichsgruppe wurden dagegen keine signifikanten Effekte auf Anhedonie und CGI beobachtet (Martinotti G et al. J Clin Psychopharmacol; im Druck).

\section{Breites Wirkprofil}

Die Wirksamkeit von Agomelatin erstrecke sich über einen breites Spektrum von Symptomen und Schweregraden, so Professor Pierre-Michel Llorca, ClermontFerrand, Frankreich. Das Antidepressivum verbessere auch Begleitsymptome der Depression wie Angst- und Schlafstörungen. Die Ergebnisse verschiedener nicht interventioneller Studien bestätigen laut Llorca die gute Wirksamkeit und Verträglichkeit auch bei heterogenen Patienten im Praxisalltag.

Abdol A. Ameri

Satellitensymposium „Challenges in the treatment of depression: the circadian approach", EPA-Kongresses, Prag, 5.3.2012; Veranstalter: Servier 